\title{
Evaluation of Flame Retardancy of Cardboards Products-Aiming for Application to Cardboard Beds in Shelters
}

\author{
Y. Mizutani' ${ }^{1}$ Y. Mochizuki', K. Nishitani' ${ }^{1}$, M. Okoshi ${ }^{2}$ \\ ${ }^{1}$ Department of Advanced Fibro-Science, Kyoto Institute of Technology, Kyoto, Japan \\ ${ }^{2}$ Organization for Research Initiatives, Yamaguchi University, Yamaguchi, Japan \\ Email: kitktkshym24@gmail.com
}

How to cite this paper: Mizutani, Y., Mochizuki, Y., Nishitani, K. and Okoshi, M. (2019) Evaluation of Flame Retardancy of Cardboards Products-Aiming for Application to Cardboard Beds in Shelters. Open Journal of Safety Science and Technology, 9, 157-167.

https://doi.org/10.4236/ojsst.2019.94011

Received: October 1, 2019

Accepted: December 10, 2019

Published: December 13, 2019

Copyright $\odot 2019$ by author(s) and Scientific Research Publishing Inc. This work is licensed under the Creative Commons Attribution International License (CC BY 4.0).

http://creativecommons.org/licenses/by/4.0/

\begin{abstract}
Japan is a country with many disasters such as earthquakes and heavy rains. Disasters cannot be prevented, however, efforts to reduce secondary damage was performed by the nation. Sleeping without beds in a shelter has a high prevalence of economy class syndrome. Due to the living environment of shelter, cots were introduced to each local government. As a result, cots were able to prevent hypothermia and freezing death. Shelters in cold regions use powerful heating devices, which lowers humidity and increases the risk of fire. Cardboard beds were made from paper so that, the user was not completely convinced of the fire risk. In this study, we investigated the combustion behavior and effects of flame-retardants on cardboard by combustion test and thermal analysis. Phosphorus-Nitrogen flame-retardant was possible to suppress the combustion which emitted a large amount of carbon monoxide.
\end{abstract}

\section{Keywords}

Cardboard, Flame-Retardant, Shelter, Disaster

\section{Introduction}

Japan is a country with a narrow land area with forests covering about $67 \%$. Land area is narrow and long with north and south and blessed with rich nature. However, nature rages and various "disasters" have attack people and torments. Disaster refers to people being affected by eruptions, earthquakes, tsunamis, floods, sediments, sometimes large-scale accidents, conflicts and wars in urban areas. In recent years, a large number of people disappeared in the Great Han- 
shin-Awaji Earthquake and the Great East Japan Earthquake. Comprehensive disaster prevention that predicts major disaster and copes with them to prevent or reduce damage has high-difficulties [1].

Concepts of reduction have been established than disaster prevention in recent years. Although the damage to the disaster occurrence is unavoidable, efforts have been made to reduce secondary damage [2]. Among them, secondary health hazards have been reported at shelters. Especially, the economy class syndrome which caused by victims sleeping in a shelter is often covered by the media since the Mid Niigata Prefecture Earthquake in 2004. Sakuma et al. investigated high morbidity of the serious economy class syndrome caused by blood clots are formed on the feet by long-term miscellaneous life in the shelter as shown in Figure 1 [3].

In April 2016, the recommending statement of a cardboard bed was included in the shelter operation guidelines established by the Cabinet Office disaster prevention department [4]. Immediately after that, on April 14 and 16, two large earthquakes occurred on Kumamoto prefecture as the epicenter and 5,300 cardboard beds were introduced on shelter as shown in Figure 2. Local governments

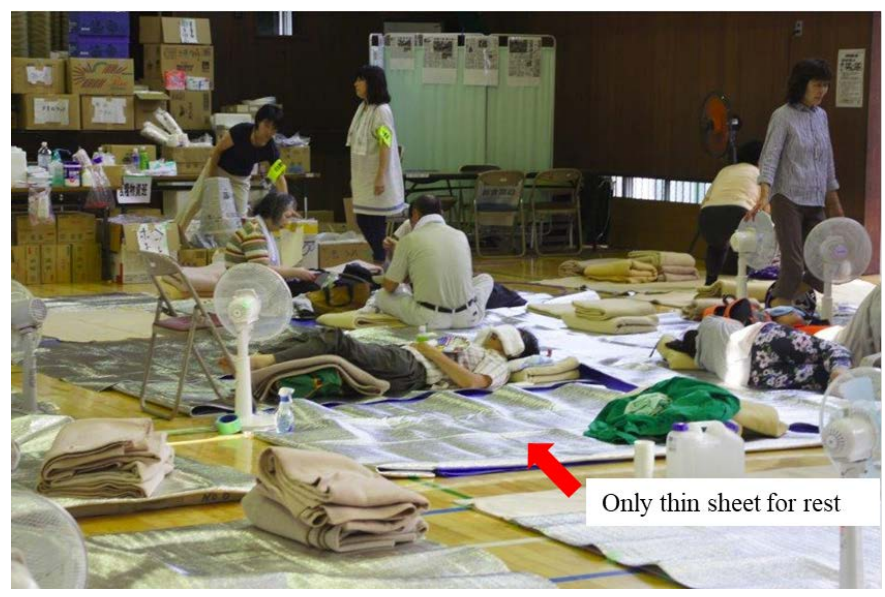

Figure 1. Conventional shelter in Japan.

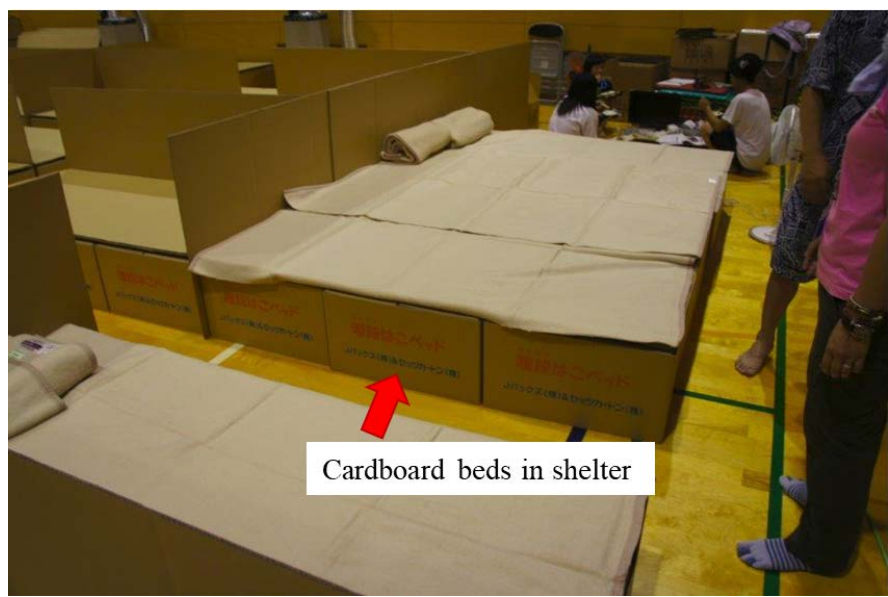

Figure 2. Cardboard bed installed shelter in Japan. 
who introduced the cardboard bed had fewer deaths related to disasters and nursing care applications than non-introduced local governments. The significant difference has been confirmed depending on the introduction of cardboard bed to shelter [5] [6].

Disaster cots such as cardboard bed and pipe bed were an overwhelming shortage of places for storing in Japan which is the small country. Cardboard beds have advantages such as capable of mass production with low-costs and produce in 72 hours. Japan has more than 2500 establishments that can be produced nationwide. Therefore, basically no need for stockpiling as an exception for some users requiring special consideration. Moreover, a cardboard bed can deliver reliably to the shelter by making the disaster prevention agreement with the government in advance [7].

Cardboard beds were made by the paper that has a high danger with the fire owing to a large number of beds are placed in a shelter. Especially, cold region shelter uses the strong heating equipment that makes low-humidity and the risk of fire becomes even higher. Cardboard beds were not having full confidence by users cause of the risk of fire.

Incombustibility of the cardboard is one of the methods to minimize the secondary damage from the fire. Incombustible is to make difficult to burn so as to reduce valuable life and property loss as much as possible and to minimize the social impact. History of incombustibility is old; the beginning is said to be B.C. and vinegar was painted on wood to prevent fire on Roman times [8]. In 1786, Geyrussac works on the incombustibility for French theater records and discovered ammonium sulfate treatment [9]. The United States military worked to make incombustibility the airplane to protect the crew and developed the halogenated paraffin and antimony oxides during World War II. These are groundbreaking flame-retardant and these were improved and are still considered as highly influential flame-retardant systems [10]. Fire is the burning that has passed the human control range and has become a massive spill condition. Combustion is a chemical reaction that involves the generation of light and heat and occurs under low pressure or similar conditions. This is basically a chemical reaction consisting of combustibles and oxygen and the highest temperature range is called the flame [11]. When the combustible substance is ignited for some reason, the temperature of gas-phase rises and the material surface heats by the radiant heat. The heat transferred into the inside of material and decomposition products were generated by reaching the decomposition temperature. The decomposition products are ejected from the material surface into the gas phase and mixed with oxygen in the gas phase so that combustible gas is generated and combustion continues. The idea that combustion can be stopped by breaking this chain process is the basic idea of incombustibility and incombustibility of combustible substances. The idea of incombustibility applies in this study and work on the incombustibility of cardboard [12].

In this study, we investigated the combustion behavior and influence of flameretardant on the cardboard to identify the problems of the cardboards. Combus- 
tion tests were performed by Multi Calorimeter and Corn Calorimeter. Five types of reagent flame-retardants and three types of commercial flame-retardants; a total of eight types of flame-retardants were selected and treated to cardboards. Optimization of the selection of flame-retardants to prevent secondary damage in shelters.

\section{Materials and Flame-Retardants}

The cardboard used W flute type (thickness $8 \mathrm{~mm}$, JPACKS Co. Ltd.) which shape is 3 sheets composition in the 3-layer structure shown in Figure 3. W flute type has the advantages of strong to heavyweights within the commercial product. Only the $\mathrm{W}$ flute type is used for the production of cardboard bed.

Five types of reagent flame-retardants and three types of commercial flameretardants were selected for the treatment of cardboard. Table 1 shows the contents of flame-retardants. P1 and P2 are organophosphorus compounds and NS is a powder compound mainly composed of nitrogen and sulfur. PNH is a compound which is liquid at normal temperature and pressure and which contains a halogen-based element in addition to phosphorus and nitrogen. $\mathrm{PN}$ is a compound of phosphorus and nitrogen with a colorless and transparent liquid. C, B, and PNS are used for general households to incombustibility a curtain, Japanese traditional shoji and tatami mat [13].

These flame-retardants have impregnated the cardboards to investigate the effects on incombustibility. Cardboards were dipped in the flame-retardants solutions for 10 minutes for impregnation. Cardboards were dried in an oven at 80 degrees after impregnation. In the case of NS, this was dissolved in water to

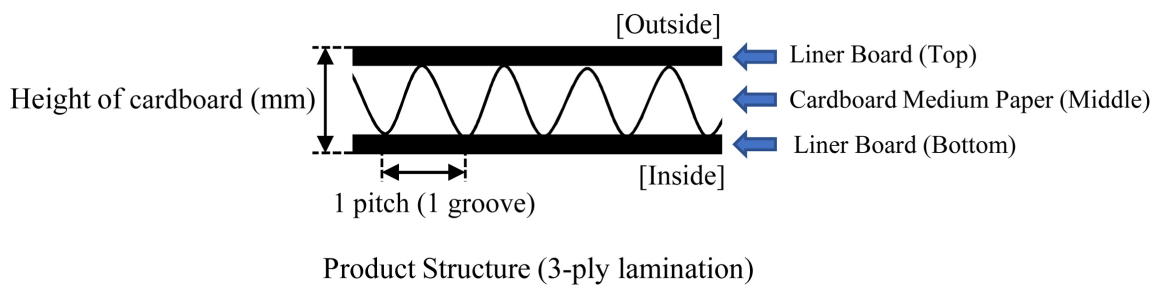

Figure 3. Structure of W-flute cardboard.

Table 1. Contains of flame-retardant.

\begin{tabular}{cccccccc}
\hline & & Phosphrus & Nitrogen & Sulfur & Halogenic & Boron & Ceremics \\
\hline & P1 & $\bullet$ & & & & & \\
Reagent & P2 & $\bullet$ & & & & & \\
Flame- & NS & & $\bullet$ & $\bullet$ & & & \\
retardant & PNH & $\bullet$ & $\bullet$ & & $\bullet$ & & \\
& PN & $\bullet$ & $\bullet$ & & & & \\
\hline $\begin{array}{c}\text { Commercial } \\
\text { Flame- } \\
\text { retardant }\end{array}$ & C & & & & & & $\bullet$ \\
& PNS & $\bullet$ & $\bullet$ & $\bullet$ & & & \\
\hline & & & & & & &
\end{tabular}


make a 50 percent aqueous solution. Table 2 shows the flame-retardants impregnation rate which calculated from the mass of the flame-retardant before and after impregnation.

\section{Test Methods}

\subsection{UL Combustion Test}

Multi Calorimeter (Toyo Seiki Seisaku-sho, Ltd.) was used for the combustion test. Multi Calorimeter has the $0.75 \mathrm{~m}^{3}$ volume draft chamber which is able to measure the heat generation rate and mass loss.

UL combustion test was performed both of vertical and horizontal direction according to ASTM D3801 and ASTM D635. This test measure and evaluate the temporal changes of heat quantity with combustion which specimen placed vertically and horizontally ignited and burned with a burner. The cardboard was cut out the size with $150 \mathrm{~mm}$ length and $15 \mathrm{~mm}$ width for vertical direction combustion test as shown in Figure 4. On the other hands, horizontal direction specimens were cut out $200 \mathrm{~mm}$ length and $50 \mathrm{~mm}$ width as shown in Figure 5. The

Table 2. Flame-retardant impregnation rate.

\begin{tabular}{cccc}
\hline & Low & Middle & High \\
\hline P1 & 30 & 44 & 50 \\
P2 & 14 & 26 & 38 \\
NS & 14 & 26 & 38 \\
PNH & 15 & 20 & 20 \\
PN & 18 & 24 & 29 \\
C & 8 & & \\
B & 9 & & \\
PNS & 9 & & \\
\hline
\end{tabular}

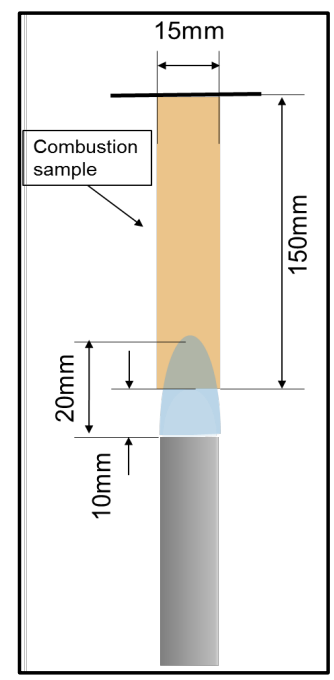

(a)

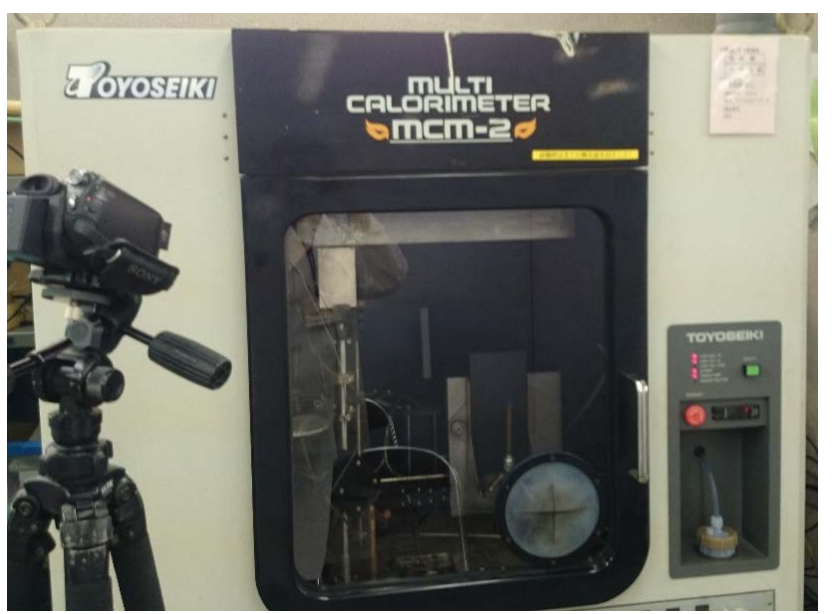

(b)

Figure 4. UL-94 Vertical test method by multi calorimeter. (a) Combustion diagram. (b) Multi calorimeter. 


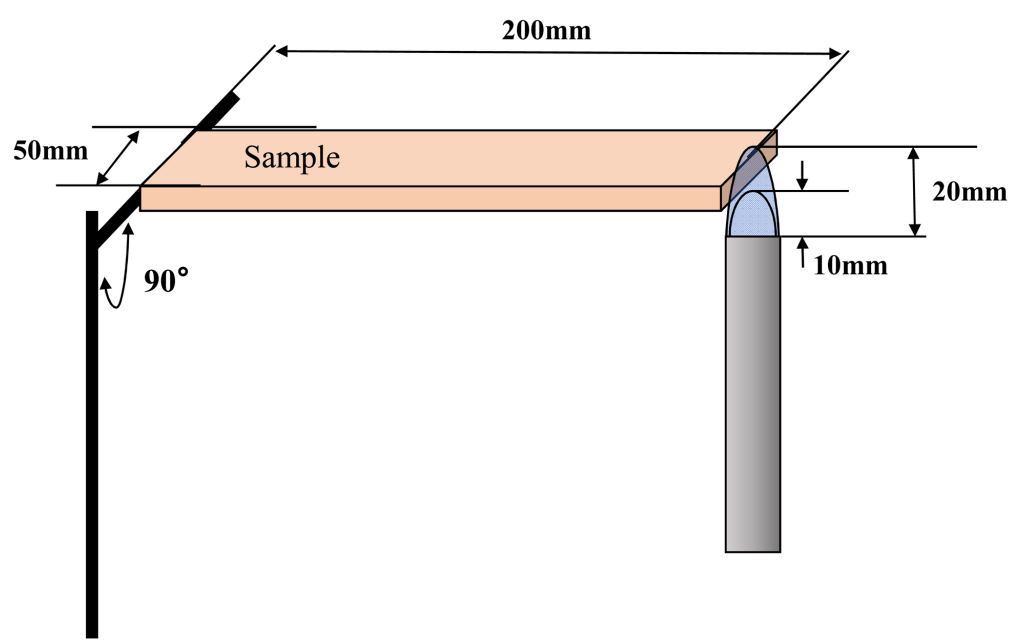

Figure 5. UL-94 Horizontal test method by multi calorimeter.

flame was set up $50 \mathrm{~W}, 20 \mathrm{~mm}$ height, and center of width with 10 seconds. Each condition was tested with three samples.

\subsection{Corn Calorimeter Test}

Corn calorimeter test was performed by Corn calorimeter C3 (Toyo Seiki Seisaku-sho Ltd.). This test measures the calorie of burning combustible gas generated by thermal decomposition with the spark which specimen placed horizontally and heated by the corn. Calorie of combustion, combustion time and time until ignition were measured in this study. Specimens were cut out with $100 \mathrm{~mm}$ square flat plate. The radiant heat of corn was set up $50 \mathrm{~kW} / \mathrm{m}^{2}$ for the measurement condition. Each specimen was tested with three samples.

\subsection{Thermal Analysis}

Thermogravimetric analysis was performed to investigate the decomposition speed of flame-retardants. Thermogravimetric analysis machine Discovery TGA (TA Instruments) was used. Flame-retardants which are P1, NS, PN, C, B and PNS, and cardboard was used for measurements. Each specimen was tested with a rising temperature of 20 degrees/minutes. Each flame-retardant was tested with three samples.

\section{Results}

\subsection{UL Combustion Test of Cardboard}

Figure 6 shows the relationship between the heat release rate (HRR) and burning time on vertical combustion test and horizontal combustion test of $\mathrm{W}$-flute cardboard. Vertical combustion has a shaper curve start and higher maximum HRR than horizontal combustion. Vertical combustion was a shorter combustion time than the horizontal combustion from the results of Figure 6. Cardboard combustion on horizontal direction took time after the ignition was observed. On the other hand, combustion of vertical direction was emitted high 


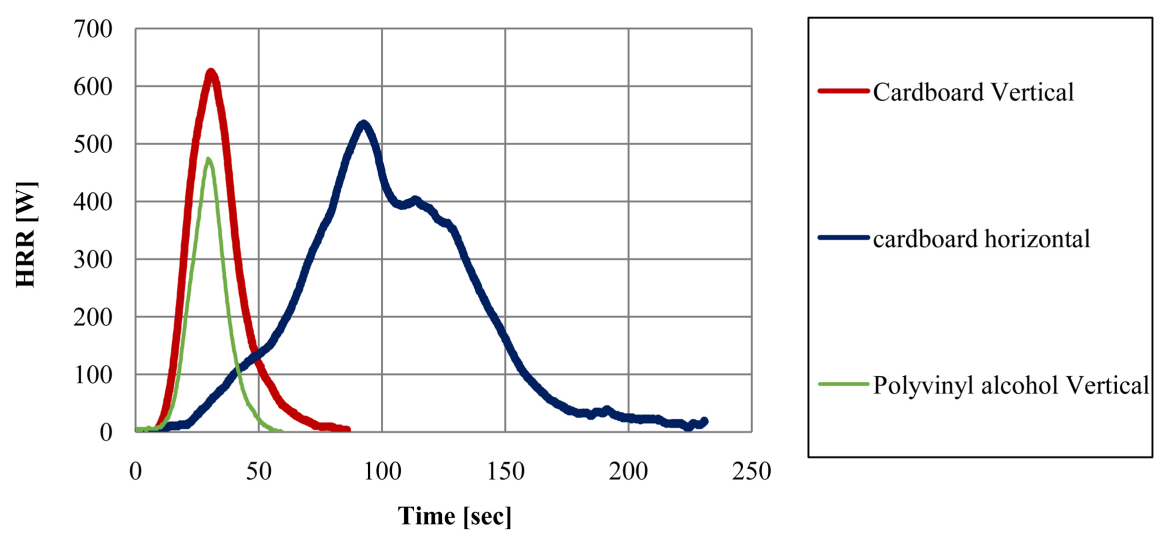

Figure 6. Grid line for analysis combustion areas.

heat immediately after ignition and burns out in a short time.

Figure 7 shows the schematic view of combustion areas in the vertical direction and horizontal direction. Combustion speed on end was faster than the center of cardboard both vertical and horizontal direction was demonstrated by these figures. In addition, combustion was spread with the shape of concentric circles stretched upward to form the fire source was observed in the center of the cardboard.

\subsection{UL Combustion Test of Flame-Retardant Cardboards}

Figure 8 shows the maximum heat release rate of each specimen according to the flame-retardant impregnation rate. Effects of Phosphorus flame-retardants $\mathrm{P} 1$ and $\mathrm{P} 2$ were small on cardboard. However, flame-retardants of PN and PNH show high flame-retardant effects which had low maximum HRR even the impregnation rate was $11 \%$ to $20 \%$. Especially, PN did not ignite the cardboard on the impregnation rate of $18 \%$. All three types of commercial flame-retardants had low impregnation rate and high maximum HRR.

\subsection{Corn Calorimeter Test}

Figure 9 shows the relationship between maximum HRR and heater temperature on flame-retardant cardboards, Relationship between total heat release (TRR) on each flame-retardant card boards and heater temperature was shown in Figure 10. P1 had a higher maximum HRR and THR than control (normal cardboard) and effects of flame-retardant were not observed. PNH and PN did not ignite when the heater temperature 475 degrees and 586 degrees, so that maximum HRR and TRR were low. The emitted carbon monoxide of flame-retardant cardboards was larger than normal cardboard when focused on Figure 11 carbon monoxide emissions during combustion.

\subsection{Thermal Analysis}

Results of the thermogravimetric analysis of flame-retardants are shown in Figure 12. The decomposition temperature of the cardboard was 300 degrees. On 


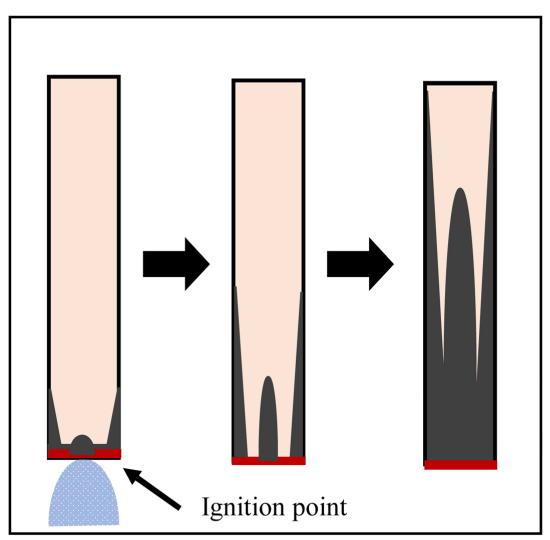

Vertical combustion test

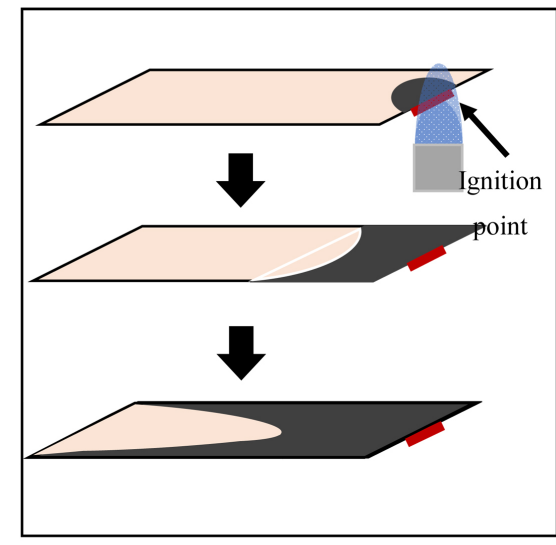

Horizontal combustion test

Figure 7. Spreading of combustion transition.

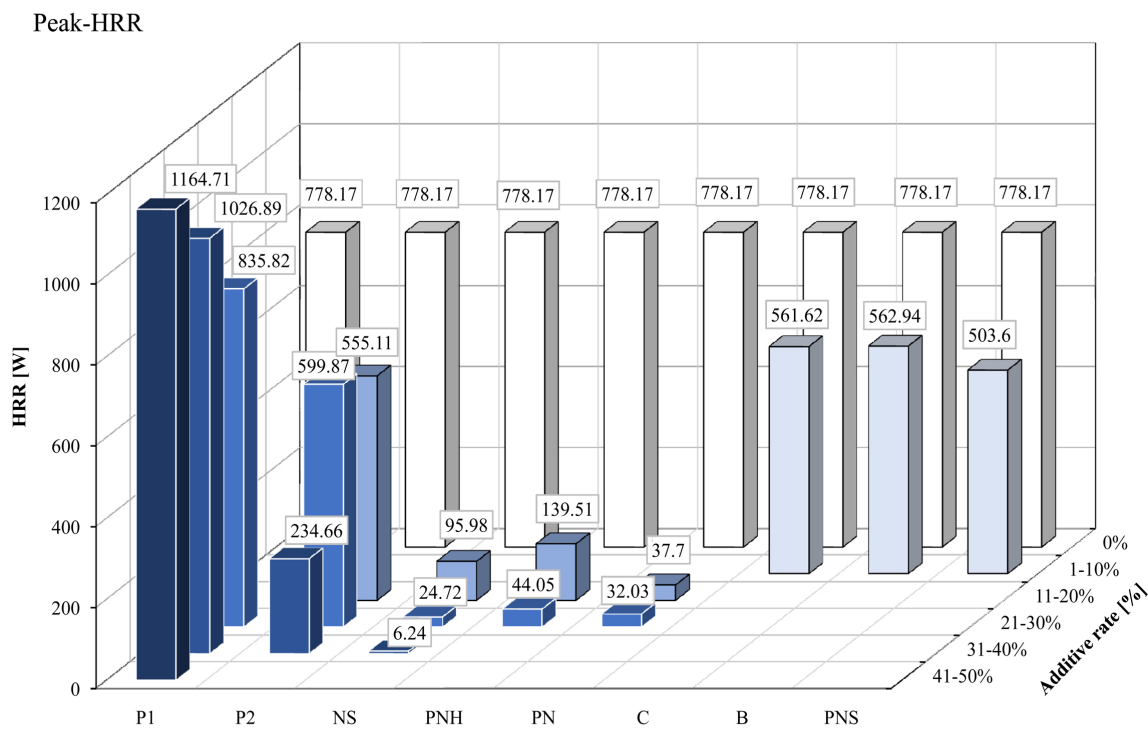

Figure 8. Peak-HRR of UL-94 vertical combustion test.

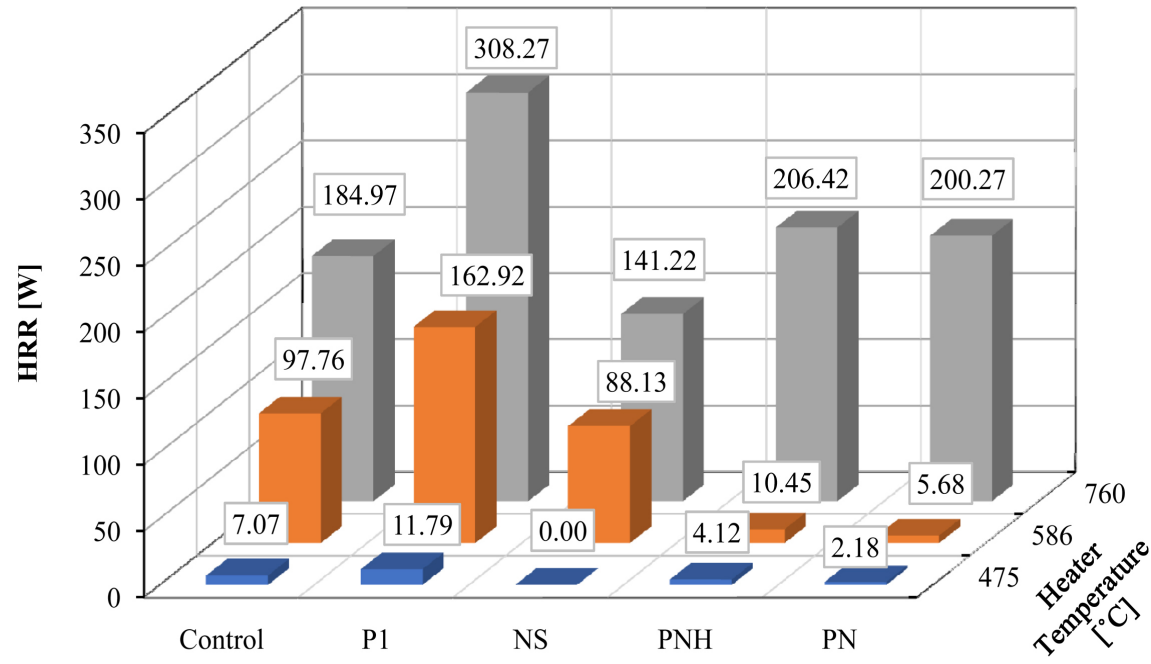

Figure 9. Relationship between peak HRR and heater temperature. 


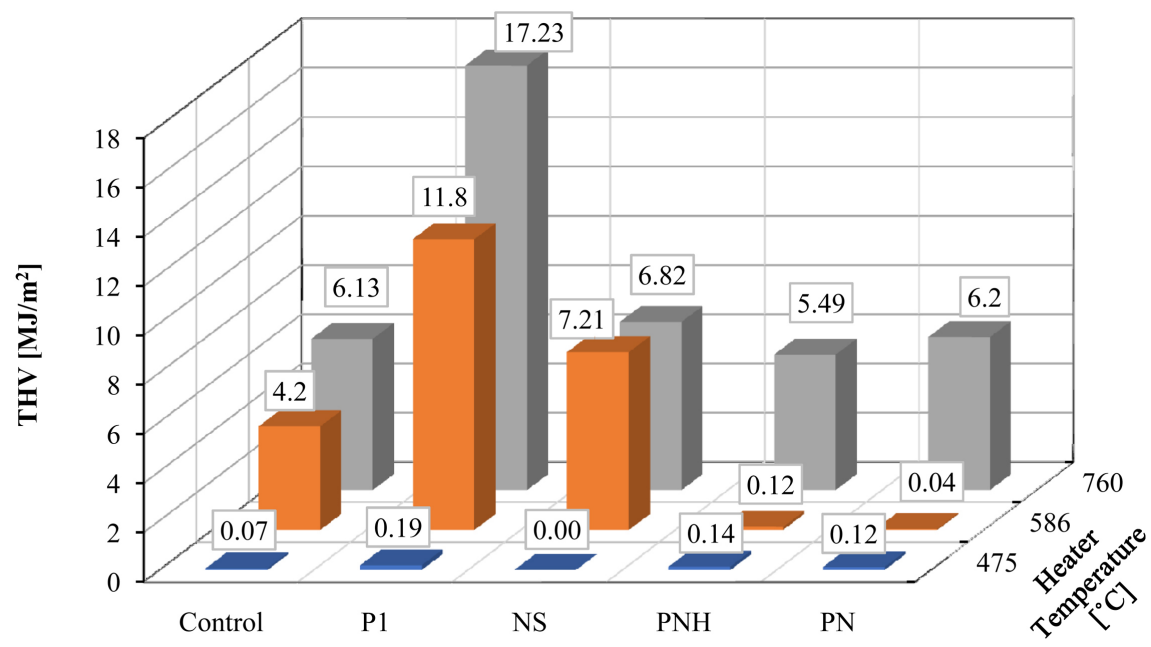

Figure 10. Relationship between THR and heater temperature.

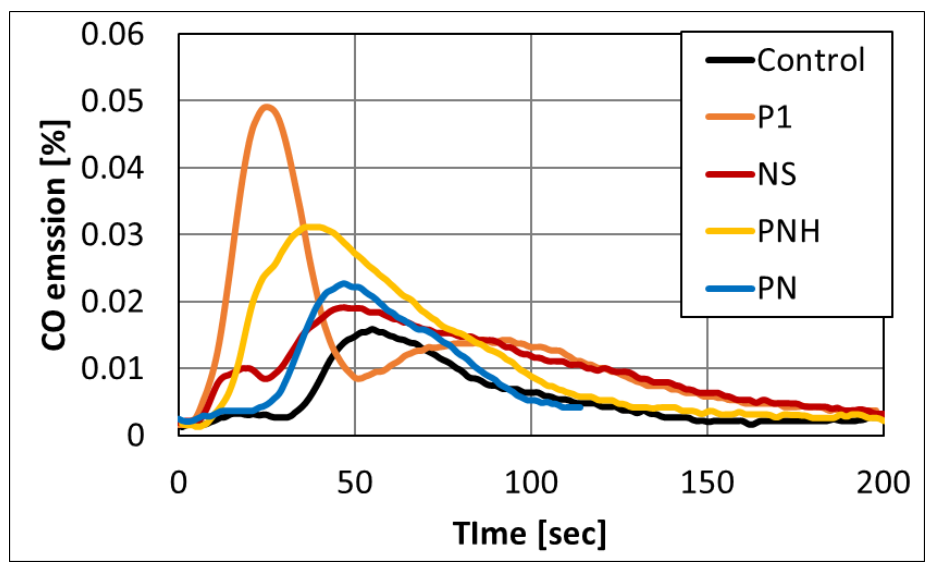

Figure 11. CO emission [\%].

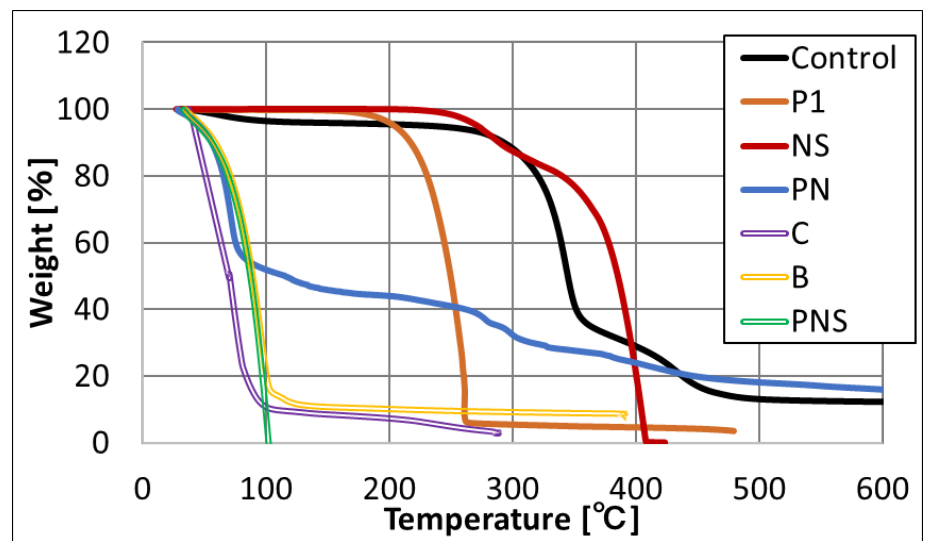

Figure 12. TGA curves of flame-retardant.

the other hand, the decomposition temperature of commercial flame-retardants which $\mathrm{C}, \mathrm{B}$, and $\mathrm{PN}$ were started from 100 degrees or less.

All specimens of residues were within $10 \%$. PN was pyrolyzed with 4 stages and residues was about $9 \%$. P1 started pyrolysis on 200 degrees and weight was 
decreased to $10 \%$ immediately. In the case of NS, pyrolysis occurred with twostages around 300 degrees and almost no residue remained.

\section{Discussion}

The compatibility between the cardboard and each flame-retardant were investigated. Regarding the commercial flame-retardants had difficulties to impregnate the cardboard and these effects were low. Pyrolysis of commercial flameretardants and phosphorus flame-retardant occurred at a lower temperature of cardboard. Therefore, these were decomposed until the cardboard combustion. So, the heat of combustion was increased on the combustion test was considered.

On the other hand, comparing the pyrolysis behavior of nitrogen-sulfur flameretardants such as $\mathrm{PN}, \mathrm{PNH}$, and the cardboard, pyrolysis occurred during the pyrolysis of the cardboard. In addition, a large amount of carbon monoxide was generated on the combustion that suppressed the combustion by emitted an inert gas from flame-retardants on combustion before and after. Flame-retardants were considered that flame-retardant effects were demonstrated in the combustion test. Form these results, we thought that PN is suitable for flame-retardant cardboard.

\section{Conclusions}

In this study, we investigated the combustion behavior and influence of flameretardants on the cardboards. UL combustion test, corn calorimeter test and thermal analysis were performed to clarify the combustion mechanism and optimization of flame-retardants.

Cardboard combusted easily in the vertical direction and combusted into a shape with concentric circle extended upward. Although commercial flame-retardants were pyrolyzed at the temperature on 100 degrees, however, PN was low in both of maximum HRR and THR and emission of carbon monoxide was high. $\mathrm{PN}$ is the suitable flame-retardant for cardboard which this emit a large amount of carbon monoxide and suppresses the combustion was clarified by these experiments.

$\mathrm{PN}$ impregnated cardboard is expected to prevent secondary damage from the fire in a shelter.

\section{Conflicts of Interest}

The authors declare no conflicts of interest regarding the publication of this paper.

\section{References}

[1] Uyeda, S. (2013) On Earthquake Prediction in Japan. Proceedings of the Japan Academy Series B, 89, 391-400.

[2] Tanaka, K. (2005) The Impact of Disaster Education on Public Preparation and Mitigation for Earthquakes: A Cross-Country Comparison between Fukui, Japan and the San Francisco Bay Area, California, USA. Applied Geography, 25, 201-225. 
https://doi.org/10.1016/j.apgeog.2005.07.001

[3] Sakuma, M., Nakamura, M., Hanzawa, K., Kobayashi, T., Kuroiwa, M., Nakanishi, N., et al. (2006) Acute Pulmonary Embolism after an Earthquake in Japan. Seminars in Thrombosis and Hemostasis, 32, 856-860.

https://doi.org/10.1055/s-2006-955468

[4] Cabinet Office, Government of Japan (2016) Sheler Management Guidelines. 7-33.

[5] Kumamoto University (2017) 4.14 4.16 Facing Difficulties beyond Expectations. 16-20. http://hdl.handle.net/2298/37381

[6] Cabinet Office, Government of Japan (2017) Report of Assistance of Victims at Shelters in 2016.

[7] Cabinet Office, Government of Japan (2013) 2013 Annual Report on Disaster Prevention.

[8] Li, A., Yang, R.Q. and Wei, H. (2011) Historical and Emerging Halogenated Flame Retardants in Sediment of the Great Lakes.

[9] Blum, A. and Ames, B.N. (1977) Flame-Retardant Additives as Possible Cancer Hazards. Science, 195, 17-23. https://doi.org/10.1126/science.831254

[10] Vladimir, T., Majid, S., Sorin, O.M. and Ferencz, S.D. (2010) Atmospheric Pressure Plasma Enhanced Synthesis of Flame Retardant Cellulosic Materials. Journal of Applied Polymer Science, 5, 281-289.

[11] Stout, H.P. (1950) Mechanism of Ignition by Local Sources of Heat. Nature, 166, 28-29. https://doi.org/10.1038/166028b0

[12] Menachem, L., Atlas, S.M. and Pearce, E.M. (1975) Flame-Retardant Polymeric Materials. Splinger, Switerland.

[13] Shigeko, N. (1982) Studies on Flame Retardant Finishing (Part 2). Journal of Home Economics of Japan. 33, 76-83. 